\title{
The Youngest Lobe-Dominated Radio Sources
}

\author{
I. Owsianik ${ }^{a, b, 1}$ J.E. Conway ${ }^{c}$ A.G. Polatidis ${ }^{c, d}$ \\ a Toruń Centre for Astronomy, ul. Gagarina 11, 87-100 Toruń, Poland \\ ${ }^{\mathrm{b}}$ Max-Planck-Institute für Radioastronomie, Auf dem Hügel 69, D-53121 Bonn, \\ Germany \\ ${ }^{\mathrm{c}}$ Onsala Space Observatory, S-43992 Onsala, Sweden \\ d Joint Institute for VLBI in Europe, Postbus 2, 7990 AA Dwingeloo, The \\ Netherlands
}

\begin{abstract}
We present an analysis of multi-epoch global VLBI observations of the Compact Symmetric Objects: $2352+495$ and $0710+439$ at $5 \mathrm{GHz}$. Analysis of data spread over almost two decades shows strong evidence for an increase in separation of the outer components of both sources at a rate of $\sim 0.2 h^{-1} \mathrm{c}$ (for $q_{\circ}=0.5$ and $H_{\circ}=100 h$ $\left.\mathrm{kms}^{-1} \mathrm{Mpc}^{-1}\right)$. Dividing the overall sizes of the sources by their separation rates implies that these Compact Symmetric Objects have a kinematic age $\ll 10^{4}$ years. These results (and those for other CSOs) strongly argue that CSOs are indeed very young sources and that they are probably evolve into the much larger classical doubles.
\end{abstract}

Key words: radio continuum: galaxies — galaxies: active — galaxies: compact — galaxies: evolution - galaxies: individual $(0710+439,2352+495)$

PACS: 98.54.Cm, 98.54.Gr, 98.62.Ai, 98.58.Ay

\section{Introduction}

There exists a class of enigmatic sources in which high luminosity radio emission regions are located 'symmetrically' on both sides of the central engine on linear scales of less than $1 \mathrm{kpc}$; the so-called Compact Symmetric Objects (Wilkinson et al., 1994). Several theories have been suggested to explain CSOs. It has been suggested that they are a young phase in the development of classical doubles (e.g., Phillips \& Mutel, 1982; Fanti et al., 1995; Readhead

I.O acknowledges financial support from OSO and JIVE 
et al., 1996b) or that they are fairly old sources in which a dense environment inhibits their growth (e.g., van Breugel et al., 1984). Finally it has been proposed that they are a separate class of short lived objects which 'fizzle out' after about $10^{4}$ years (Readhead et al., 1994). An obvious way to try to distinguish between these models is to investigate the growth in overall sizes of CSOs and hence set limits to their ages directly.

\section{Data analysis for $0710+439$}

The radio source $0710+439(z=0.518$, Lawrence et al., 1996) has a high radio luminosity $\left(L_{5 \mathrm{GHz}}=5 \times 10^{26} h^{-2} \mathrm{~W} \mathrm{~Hz}^{-1}\right.$, Readhead et al., 1996a). The radio flux density is very weakly polarised and it is not time variable (Aller et al., 1992).
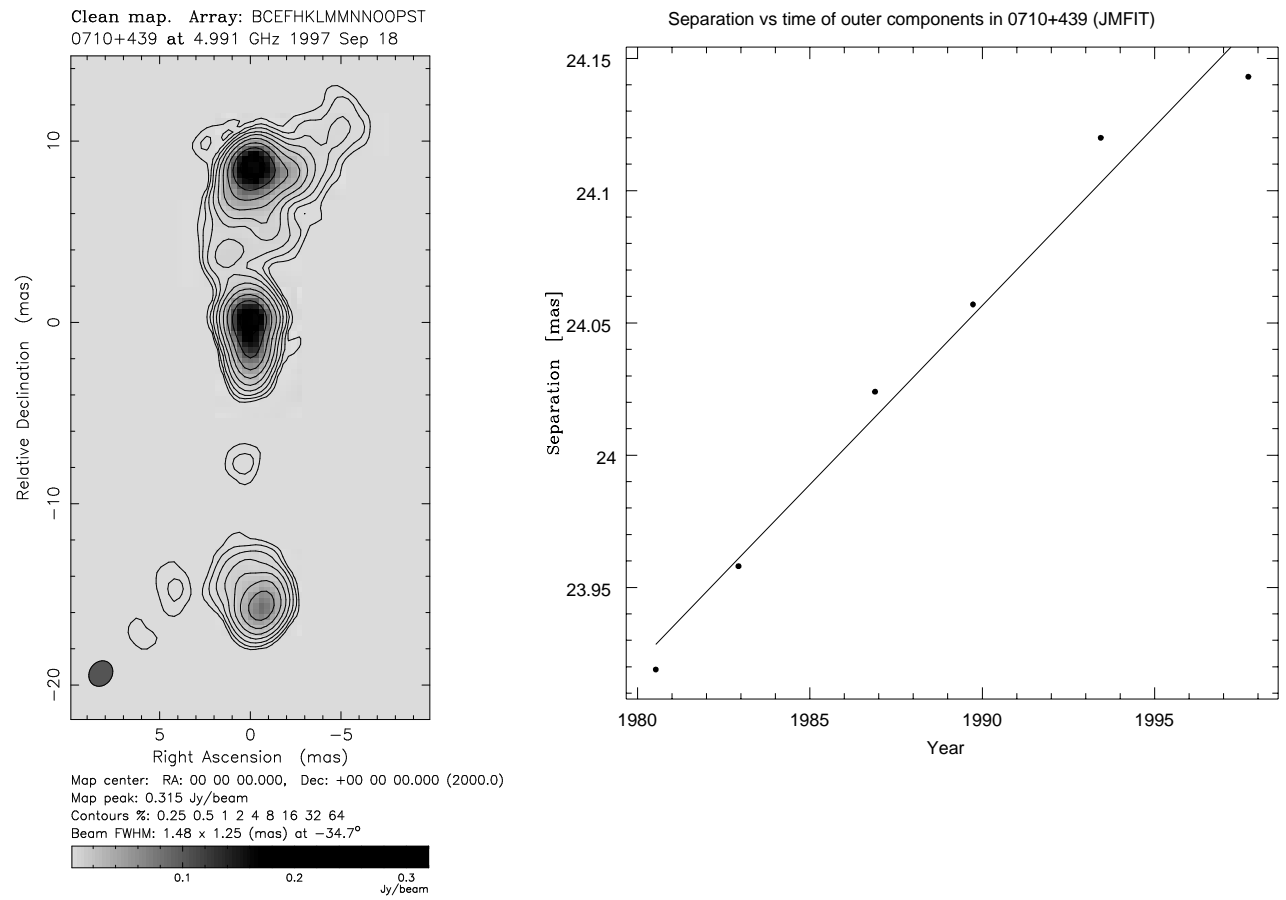

Fig. 1. (a) The natural weighted clean image of $0710+439$ at $5 \mathrm{GHz}$ from the epoch 1997.71, rms noise $151 \mu \mathrm{Jy}_{\text {beam }}{ }^{-1}$, (b) Changes in separation with time between the hotspots. Dots represent data obtained by JMFIT, solid line shows linear regression fit. See text for a discussion of the errors.

0710+439 has been observed at $5 \mathrm{GHz}$ with a global VLBI array at 6 epochs spread over a period of 17 years. The first five epochs were analysed by Owsianik \& Conway (1998). Here we add new data from multi-snapshot 14 station global observations made at 18th of Sep 1997. The highest dynamic range image (see Fig. 1a) was obtained using DIFMAP (Shepherd et al., 1994) and was used as a starting point in re-mapping the other epochs following the procedure describe by Owsianik \& Conway (1998). This image shows clearly the 
overall triple structure of the source. The northern and the southern components show compact, bright subcomponents which are associated with hotspots surrounded by the faint extended lobe emission. The middle component is associated with the base of the northern jet, and the centre of activity lies at the southern end of this component (Taylor et al., 1996). The image in Fig. 1a shows clearly for the first time at this frequency a bridge of emission between northern hotspot and the middle component. In addition the emission previously detected at $1.6 \mathrm{GHz}$ (Xu, 1994) between the middle and southern components is for the first time now also detected at $5 \mathrm{GHz}$.

The hotspot components are well separated on CLEAN images which allows us to use the AIPS task JMFIT to fit the position of hotspot at each epoch. The fitted linear regression line to this data gives an estimated separation rate of $13.614 \pm 0.988 \mu \mathrm{as} / \mathrm{yr}$ (Fig. 1b). From the obtained correlation coefficient of 0.989 we can reject the null hypothesis of no motion at a better than $0.1 \%$ confidence level (for a fuller discussion on errors see Owsianik \& Conway, 1998). Dividing the distance between hotspots of $87.07 h^{-1}$ pc by their observed separation velocity of $0.243 \pm 0.018 h^{-1} \mathrm{c}$ we estimate that the source $0710+439$ is $1100 \pm 100$ years old.

Assuming that hotspots are close to their equipartition pressure and assuming that the source is orientated not too far from the sky plane (as supported by the source having an arm-length ratio close to one) then ram pressure arguments imply an external density of $2 h^{18 / 7} \mathrm{~cm}^{-3}$. From the velocity of advance of $0710+439$ we can calculate that the rate of work done advancing the two hotspots is $5 \times 10^{43} h^{-17 / 7} \mathrm{erg} \mathrm{s}^{-1}$. Following the arguments of Readhead et al. (1996a) we can estimate an upper limit on the total jet power of $4 \times$ $10^{44} h^{-10 / 7} \mathrm{erg} \mathrm{s}^{-1}$. A lower limit on the jet power equals the sum of work of advance and radio luminosity $\left(7 \times 10^{43} h^{-2} \mathrm{erg} \mathrm{s}^{-1}\right)$. Given these numbers we can estimate that the efficiency $(\epsilon)$ of conversion of jet mechanical energy into radio emission in $0710+439$ is $23 \%<\epsilon<53 \%$ (for $h=0.6$ ).

\section{Data analysis for $2352+495$}

The radio source $2352+495(z=0.238$, Lawrence et al., 1996) has a high radio luminosity ( $L_{5 \mathrm{GHz}}=1 \times 10^{26} \mathrm{~h}^{-2} \mathrm{~W} \mathrm{~Hz}^{-1}$, Readhead et al., 1996a). The radio flux density is very weakly polarised and it does not exhibit a significant time variation (Aller et al., 1992). Conway et al. (1992) revealed for the first time the triple radio morphology of $2352+495$ and from analysis of multiepoch data it was argued that the center of activity was associated with the middle component (Conway et al., 1992). Later $15 \mathrm{GHz}$ observations located a compact core component at the southern end of this middle component (Taylor et al., 1996). 

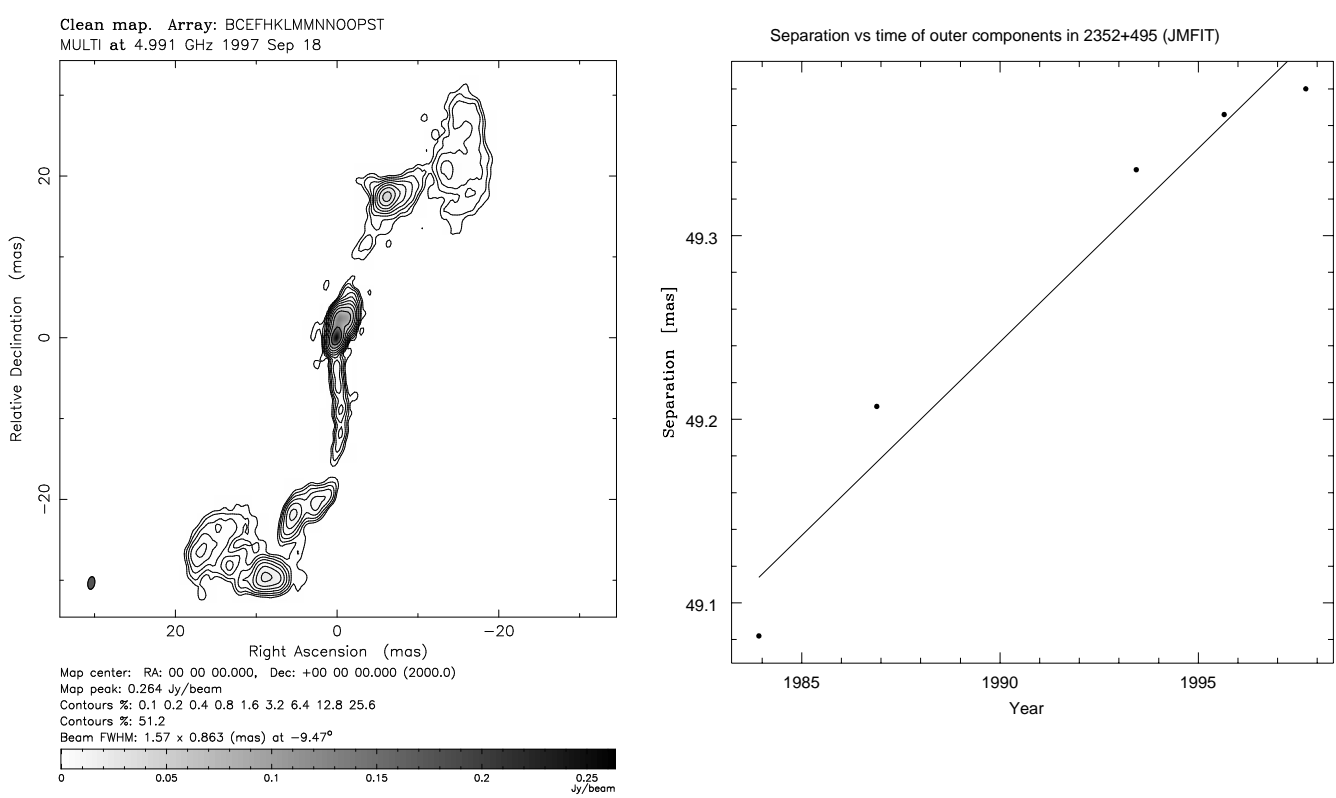

Fig. 2. (a) Natural weighted clean image of $2352+495$ at $5 \mathrm{GHz}$ from the epoch 1997.71, rms noise $57 \mu J y$ beam $^{-1}$, (b) Changes in separation with time between hotspots obtained by JMFIT, solid line shows linear regression fit. See text for a discussion of the errors.

$2352+495$ has been observed at $5 \mathrm{GHz}$ with a global VLBI array at five epochs spread over a period of 14 years. The first two epochs included in our analysis were made at epoch 1983.93 with a global array of 6 telescopes and then at epoch 1986.89 using multiple snapshots with 9 telescopes (see Conway et al., 1992). Here we reanalyse these epochs and add data from three additional epochs; a multi snapshot 12 station global VLBI observations made at epoch 1993.44, a 10 station VLBA observations made at epoch 1995.67 (donated by G.B. Taylor and R.C. Vermuelen, gratefully acknowledged by the authors) and finally a 14 station global VLBI observations made at epoch 1997.71. The telescopes used included those from the European VLBI Network (EVN), the Very Long Baseline Array (VLBA), the Very Large Array (VLA) and Haystack Observatory.

The analysis of the data followed the procedure described in Owsianik \& Conway (1998). Fig. 2a shows the best image of $2352+495$ obtained for the last epoch data using DIFMAP (Shepherd et al., 1994), this map was used as a starting point in analysis of the earlier epochs. This 5th epoch map clearly shows the overall triple structure of the source. The northern and the southern components show compact, bright subcomponents which are associated with hotspots surrounded by fainter extended emission from the lobes. The middle very bright component appears to be associated with the base of the northern jet (Taylor et al., 1996). The image also shows an almost continuous jet connecting the middle component with the southern hotspot. For the first time this image also reveals portions of a possible jet-like component on the 
other side of the middle component/core feature.

The well separated hotspot components in the CLEAN images allowed us to use the AIPS task JMFIT to fit the position of hotspots at each epoch. The linear regression fits to the observed changes in gaussian component separation gave us an estimate of the relative angular separation rate of $21.062 \pm$ $2.704 \mu \mathrm{as} / \mathrm{yr}$ corresponding to a separation velocity of $0.202 \pm 0.026 h^{-1} \mathrm{c}(\mathrm{Fig}$. $2 \mathrm{~b})$. The linear regression fit gives a correlation coefficient of 0.976 which allows us to reject the null hypothesis of no motion at the better than $1 \%$ confidence level. Dividing the distance between hotspots of $117.03 h^{-1}$ pc by their observed separation rate we estimate that $2352+495$ is $1900 \pm 250$ years old.

Assuming that hotspots are close to their equipartition pressure and assuming that the source is orientated not too far from the sky plane (consistent with an arm-length ratio very close to 1) then ram pressure arguments imply an external density of $1 h^{18 / 7} \mathrm{~cm}^{-3}$, which is consistent with the NLR intercloud medium. Given our estimate of an age and the jet thrust we can compare the power of advance required to drive the hotspot forward, with the radio luminosity and the jet power (Readhead et al., 1996a). For an age of $2352+495$ the rate of work done in advancing the two hotspots is $1 \times 10^{43} h^{-17 / 7} \mathrm{erg} \mathrm{s}^{-1}$. The upper limit on the total power supplied by the jet is $8 \times 10^{43} h^{-10 / 7} \mathrm{erg} \mathrm{s}^{-1}$ (Readhead et al., 1996a). The lower limit on the total power of the jet is the sum of the power of advance and the radio luminosity $\left(3 \times 10^{42} h^{-2} \mathrm{erg} \mathrm{s}^{-1}\right)$. Given these numbers we can therefore estimate that the efficiency of conversion of the jet mechanical energy into the radio emission is $5 \%<\epsilon<19 \%$ (for $h=0.6)$.

\section{Evolution of CSOs}

In addition to the two sources presented in this paper there are now reliable velocities of expansion for three other CSOs; namely 0108+388 (Owsianik et al., 1998), 2021+614 (Conway et al. (1994) and Tschager et al., this volume) and 1943+456 (Polatidis, this volume). All of the expansion velocities are of order $0.2 h^{-1} \mathrm{c}$; giving hotspot advance speeds of an order $0.1 h^{-1} \mathrm{c}$ and ages of a few thousand years. It has therefore become clear that the majority of CSOs are very young sources, which grow fairly rapidly Owsianik \& Conway, 1998; Owsianik et al., 1998). What is still uncertain is their subsequent evolution. The simplest scenario is that CSOs evolve via Medium-size Symmetric Objects into Large-size Symmetric Objects (Fanti et al., 1995; Readhead et al. 1996b; Owsianik \& Conway, 1998). Given the rapid expansion rate of CSOs the sources will spend only a short time in the CSO phase, and it would be expected that only a small fraction of sources would be CSOs. In fact CSOs 
comprise about $7.5 \%$ of sources in the flux limited surveys at $5 \mathrm{GHz}$ (Polatidis, this volume). However, this large fractional population can be explained if, as is theoretically expected, there is a strong negative luminosity evolution with increasing size. In such a model CSOs will evolve into more numerous, but much weaker sources (e.g., Begelman, 1996; Readhead et al., 1996b; Owsianil \& Conway, 1998). As noted by Readhead et al. (1996a) for $2352+495$, and as we find here the limits on radiative efficiency for CSOs compared to 'classical' large lobe-dominated sources (in which $\epsilon$ is of order of few per cent Owsianik \& Conway, 1998) empirically demonstrates that the expected luminosity evolution does in fact occur and with a size consistent with that expected by theoretical models (Begelman, 1996; Readhead et al., 1996a).

\section{References}

Aller, M.F., Aller, H.D. \& Hughes, P.A., 1992, ApJ, 399, 16.

Begelman, M.C., 1996, in: Carilli, C.L. \& Harris, D.E. (eds), Cygnus A -Study of a radio galaxy, Cambridge University Press, p. 209.

Conway, J.E., Pearson, T.J., Readhead, A. C.S., Unwin, S.C., Xu, W. \& Mutel, R.M., 1992, ApJ, 396, 62.

Conway, J.E., Myers, S.T., Pearson, T.J., Readhead, A. C.S., Unwin, S.C. \& Xu, W. 1994, ApJ, 425, 568.

Fanti, C., Fanti, R., Dallacasa, D., Schilizzi, R.T., Spencer, R.E. \& Stanghellini, C., 1995, A\&A, 302, 317.

Lawrence, C.R., Zucker, J.R., Readhead, A. C.S., Unwin, S., Pearson, T. \& Xu, W., 1996, ApJS, 107, 541.

O’Dea, C.P. \& Baum, S.A., 1997, AJ, 113, 148.

Owsianik, I. \& Conway, J.E., 1998, A\&A, 337, 69.

Owsianik, I., Conway, J.E. \& Polatidis, A.G., 1998, A\&A, 336, L37.

Phillips, R.B. \& Mutel, R.L., 1982, A\&A, 106, 21.

Readhead, A. C.S., Xu, W., Pearson, T.J., Wilkinson, P.N. \& Polatidis, A.G., 1994,in: Zensus, J. A. \& Kellermann, K. I. (eds), Compact Extragalactic Radio Sources, NRAO, p. 17.

Readhead, A. C.S., Taylor, G.B., Xu, W., Pearson, T.J., Wilkinson, P.N. \& Polatidis, A.G., 1996a, ApJ, 460, 612.

Readhead, A. C.S., Taylor, G.B., Pearson, T.J. \& Wilkinson, P.N., 1996b, ApJ, 460, 634.

Reynolds, C.S. \& Begelman, M.C., 1997, ApJ, 487, L135.

Shepherd, M. C.,Pearson T. J., Taylor G. B., 1994, BAAS, 26, 987.

Taylor, G.B., Readhead, A.C.S. \& Pearson, T.J., 1996, ApJ, 463, 95.

van Breugel, W. J.M., Miley, G. \& Heckman, T., 1984, AJ, 89, 5.

Wilkinson, P.N., Polatidis, A.G., Readhead, A.C.S., Xu, W. \& Pearson, T.J., 1994, ApJ, 432, L87.

Xu, W., 1994, PhD thesis, California Institute of Technology. 